\title{
2-Aminoethoxydiphenylborate (2-APB) inhibits release of phosphatidylserine-exposing extracellular vesicles from platelets
}

\author{
Hao Wei ${ }^{1}$, Jessica E. Davies ${ }^{1}$ and Matthew T. Harper ${ }^{1}$
}

\begin{abstract}
Activated, procoagulant platelets shed phosphatidylserine (PS)-exposing extracellular vesicles (EVs) from their surface in a $\mathrm{Ca}^{2+}$ - and calpain-dependent manner. These PS-exposing EVs are prothrombotic and proinflammatory and are found at elevated levels in many cardiovascular and metabolic diseases. How PS-exposing EVs are shed is not fully understood. A clearer understanding of this process may aid the development of drugs to selectively block their release. In this study we report that 2-aminoethoxydiphenylborate (2-APB) significantly inhibits the release of PSexposing EVs from platelets stimulated with the $\mathrm{Ca}^{2+}$ ionophore, $\mathrm{A} 23187$, or the pore-forming toxin, streptolysin-O. Two analogues of 2-APB, diphenylboronic anhydride (DPBA) and 3-(diphenylphosphino)-1-propylamine (DP3A), inhibited PS-exposing EV release with similar potency. Although 2-APB and DPBA weakly inhibited platelet PS exposure and calpain activity, this was not seen with DP3A despite inhibiting PS-exposing EV release. These data suggest that there is a further target of 2-APB, independent of cytosolic $\mathrm{Ca}^{2+}$ signalling, PS exposure and calpain activity, that is required for PS-exposing EV release. DP3A is likely to inhibit the same target, without these other effects. Identifying the target of 2-APB, DPBA and DP3A may provide a new way to inhibit PS-exposing EV release from activated platelets and inhibit their contribution to thrombosis and inflammation.
\end{abstract}

\section{Introduction}

Platelets are the main cellular component of haemostasis. They adhere, activate and aggregate at sites of vascular injury, forming a haemostatic plug that prevents blood loss. However, platelet activation on a ruptured coronary atherosclerotic plaque is the main event in coronary thrombosis, leading to unstable angina or myocardial infarction ${ }^{1}$. Anti-platelet drugs are therefore used to prevent coronary thrombosis in at-risk patients ${ }^{2,3}$.

During platelet activation, procoagulant platelets expose phosphatidylserine (PS) in the outer leaflet of their plasma membrane. PS is normally restricted to the inner leaflet by an aminophospholipid translocase ('flippase'). A high, sustained increased in cytosolic $\mathrm{Ca}^{2+}$ concentration $\left(\left[\mathrm{Ca}^{2+}\right]_{\text {cyt }}\right)$ leads to inhibition of the flippase and activation of a non-selective phospholipid scramblase,

\footnotetext{
Correspondence: Matthew T. Harper (mth29@cam.ac.uk)

'Department of Pharmacology, University of Cambridge, Cambridge, UK Edited by I. Amelio
}

TMEM16 $\mathrm{F}^{4-6}$. The result is net movement of PS to the outer leaflet. PS forms a procoagulant surface for the tenase and prothrombinase coagulation complexes, increasing thrombin generation ${ }^{7-9}$.

Procoagulant platelets also release PS-exposing extracellular vesicles (EVs). These are prothrombotic and proinflammatory ${ }^{10,11}$. The levels of PS-exposing EVs are increased in many cardiovascular and metabolic disorders $^{12-17}$, and platelets are the major source of circulating PS-exposing EVs ${ }^{11,18}$. The pathological role of PSexposing EVs, and their elevation in a range of diseases, makes them an attractive therapeutic target.

How PS-exposing EVs are shed from the plasma membrane is not fully understood. A clearer understanding of this process may aid the development of drugs to selectively block their release. PS exposure itself may be important, as they are not shed from platelets from Tmem $16 f^{-/}$mice or from patients with Scott Syndrome, a very rare bleeding disorder caused by 
lack of functional TMEM16F $\mathrm{F}^{5,19}$. In addition, high $\left[\mathrm{Ca}^{2+}\right]_{\mathrm{cyt}}$ in procoagulant platelets activates the $\mathrm{Ca}^{2+}$-dependent protease, calpain ${ }^{20,21}$. Calpaindependent cleavage of cytoskeletal proteins is also necessary. However, little is known of the mechanism beyond these events. Indeed, it is not clear whether any events other than PS exposure and calpain activity are required downstream of increased $\left[\mathrm{Ca}^{2+}\right]_{\mathrm{cyt}}$.

Platelets can be effectively stimulated to shed PSexposing EVs by $\mathrm{Ca}^{2+}$ ionophores, such as A23187, triggering a high, sustained increase in $\left[\mathrm{Ca}^{2+}\right]_{c y t}$. Although not a physiological stimulus, by bypassing platelet receptors and their proximal signalling cascades it can be used to understand the downstream mechanisms of EV release. PS-exposing EVs can be distinguished by their size and their capacity to bind annexin V (AnV). During preliminary experiments, we were surprised to find that 2aminoethoxydiphenylborate (2-APB) significantly inhibited A23187-induced annexin $\mathrm{V}$-positive $\left(\mathrm{AnV}^{+}\right) \mathrm{EV}$ release. This was surprising since $2-\mathrm{APB}$ is a cellpermeable modulator of numerous ion channels, particularly $\mathrm{Ca}^{2+}$ channels, yet we expected the use of a $\mathrm{Ca}^{2+}$ ionophore to bypass any contribution of plasma membrane or intracellular $\mathrm{Ca}^{2+}$ channels. Therefore, in this study, we investigated the mechanism of how 2-APB inhibits the release of $\mathrm{AnV}^{+} \mathrm{EVs}$ from platelets. We find that the effect of 2-APB does not depend on inhibition of $\mathrm{Ca}^{2+}$ signalling, PS exposure or calpain activity. These results indicate that events in addition to PS and calpain activation are required for release of PS-exposing EVs and suggest the existence of other potential targets to inhibit this important pathological process.

\section{Results}

\section{2-APB inhibits $\mathrm{AnV}^{+}$EV release induced by the $\mathrm{Ca}^{2+}$ ionophore, A23187}

In order to better understand the processes by which platelets release PS-exposing EVs, platelets were stimulated with the $\mathrm{Ca}^{2+}$ ionophore, A23187. This triggers rapid $\mathrm{Ca}^{2+}$ entry, resulting in PS exposure and release of PS-exposing EVs. PS exposure was detected using annexin $\mathrm{V}$ (AnV) (Fig. 1a). AnV ${ }^{+}$platelets and $\mathrm{AnV}^{+}$EVs were detected by flow cytometry. As we have previously discussed, this approach is likely to preferentially detect the largest $\mathrm{AnV}^{+} \mathrm{EVs}$ and to underestimate the total number of EVs released ${ }^{22}$. However, it is still a rapid and effective means of assessing $\mathrm{AnV}^{+} \mathrm{EV}$ release.

Pre-treatment with 2-APB $(100 \mu \mathrm{M})$ significantly inhibited $\mathrm{AnV}^{+} \mathrm{EV}$ release compared to platelets treated with the vehicle (DMSO) at all concentrations of A23187 tested. In contrast, the percentage of $\mathrm{AnV}^{+}$platelets was only weakly affected (Fig. 1a-c). When $10 \mu \mathrm{M}$ A23187 was used, 2-APB inhibited $\mathrm{AnV}^{+} \mathrm{EV}$ release with a $\mathrm{pIC}_{50}$ of $4.45 \pm 0.09$ ( $\mathrm{IC}_{50}$ approximately $35 \mu \mathrm{M} ; n=5$ ), with no corresponding effect on platelet $\mathrm{AnV}^{+}$binding (Fig. 1d).

To assess the reversibility of the inhibition, platelets were treated with $2-\mathrm{APB}$ or DMSO then washed by centrifugation. The procedure of washing platelets by centrifugation resulted in slightly greater sensitivity to A23187 $\left(\mathrm{pEC}_{50}\right.$ of $5.77 \pm 0.20$ for $\mathrm{AnV}^{+} \mathrm{EV}$ release prior to washing; $\mathrm{pEC}_{50}$ of $6.16 \pm 0.12$ after washing). 2-APBtreated platelets released significantly fewer $\mathrm{AnV}^{+} \mathrm{EVs}$ when stimulated with A23187. Notably, there was now no difference in platelet AnV binding between 2-APB-treated and DMSO-treated platelets at any concentration of A23187 (Supplementary Fig. 1).

Together, these data indicate that 2-APB inhibits A23187-induced $\mathrm{AnV}^{+}$EV release. This action not related to the weak inhibition of platelet PS exposure.

\section{Structural analogues of 2-APB show differing effects on $\mathrm{AnV}^{+} \mathrm{EV}$ release}

2-APB has many reported targets. To further characterise its effects, we used a small panel of related, commercially available compounds (Supplementary Fig. 2). The effects of these analogues on some 2-APB targets has been previously described (Supplementary Table 1).

Some analogues had no effect on A23187-induced AnV ${ }^{+}$ EV release (Fig. 2). Phenylborinic acid (PBA) has the boron atom attached to only one phenyl group and may be too simple a fragment to effectively inhibit $\mathrm{AnV}^{+} \mathrm{EV}$ release. Since di-PBA is unstable ${ }^{23}$, we used the more stable analogue, dimesitylborinic acid (DMBA), which has two phenyl rings replaced by mesityl groups. This was also ineffective at $100 \mu \mathrm{M}$. It may be that analogues with a terminal $\mathrm{B}-\mathrm{OH}$ are ineffective and that a $\mathrm{B}-\mathrm{O}$ core is required.

Two analogues that lack the boron atom were also ineffective: 2,2-diphenyltetrahydrofuran (DPTHF), which has a five-membered ring containing an oxygen atom, similar to the ring form of 2-APB, but lacks a boron or nitrogen atom, and diphenhydramine (DPHD), which is similar to the linear form of 2-APB, but lacks the boron atom and has two methyl groups attached to the secondary amine.

In contrast, diphenylboronic anhydride (DPBA) and 3(diphenylphosphino)-1-propylamine (DP3A) both inhibited $\mathrm{AnV}^{+} \mathrm{EV}$ release. Both were of similar potency to 2APB. The $\mathrm{pIC}_{50}$ for DPBA was $4.55 \pm 0.32 \quad\left(\mathrm{IC}_{50}\right.$ approximately $28 \mu \mathrm{M} ; n=5$ ). The $\mathrm{pIC}_{50}$ for DP3A was $4.38 \pm 0.14 \quad\left(\mathrm{IC}_{50}\right.$ approximately $\left.42 \mu \mathrm{M} ; n=5\right)$. The effectiveness of DPBA and DP3A indicates that neither the amine group nor the boron atom is necessary for inhibition. In addition, DPBA partially reduced the percentage of $\mathrm{AnV}^{+}$platelets, whereas DP3A did not, suggesting that the boron atom may be important for this weak inhibition. 


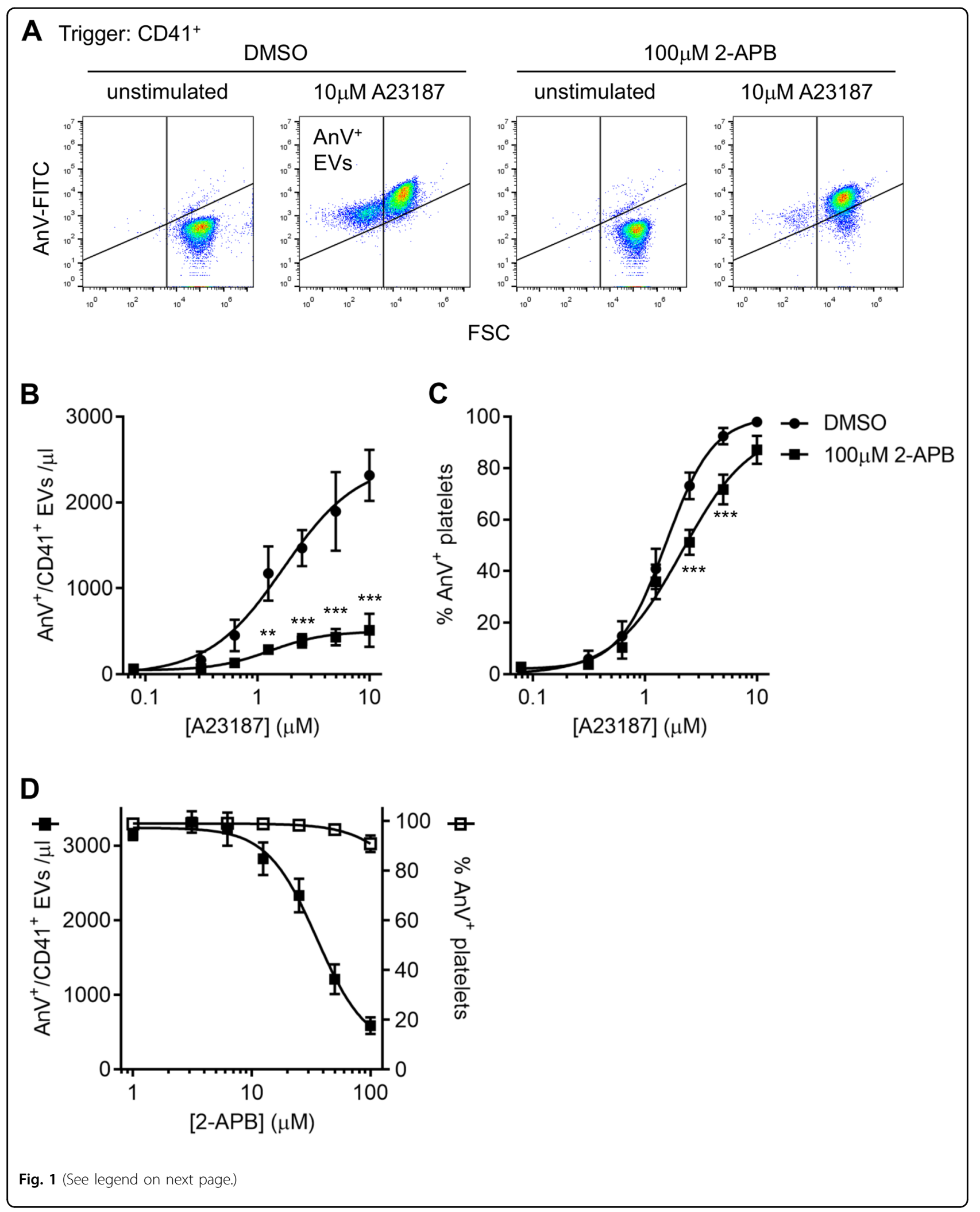


(see figure on previous page)

Fig. 1 2-APB inhibits release of PS-exposing ( $\mathbf{A n V}^{+}$) extracellular vesicles (EVs). a Washed platelets were treated with 2-APB (100 $\mu$ M; $\left.30 \mathrm{~min}\right)$ or the solvent, DMSO, as control, then stimulated with the indicated concentration of A23187 for 10 min, after which samples were stained with antiCD41a-PerCP-Cy7, and annexin V-FITC to detect PS exposure. PerCP-Cy7 fluorescence was used to trigger acquisition of CD41 ${ }^{+}$events. The panels show density plots of events from low density (blue) to high density (red) of forward scatter (FSC) and FITC fluorescence. Unstimulated platelets have high FSC-A and low annexin V-FITC binding (LR). Stimulation with A23187 triggered PS exposure in platelets and release of PS-exposing (AnV ${ }^{+}$) EVs. The vertical line separating left and right was defined by the FSC of $1 \mu \mathrm{m}$ silica beads. The density plots are representative of data from five different donors. b, c Platelets were stimulated with the indicated concentration range of A23187. Mean data ( \pm s.e.m.; $n=5)$ are shown for $A n V^{+} E V s(\mathbf{b})$ and the percentage of platelets $(>1 \mu \mathrm{m})$ binding AnV. Concentration-response curves were fitted with a four-parameter logistic equation. Data were further analysed by two-way RM-ANOVA with Sidak's post-test. ${ }^{* *} p<0.01 ;{ }^{* * *} p<0.001(n=5)$. d Platelets were treated with a range of concentrations of 2-APB prior to stimulation with $10 \mu \mathrm{M} \mathrm{A23187}(n=5)$.

The inhibitory effect of 2-APB is not through inhibition of $\mathrm{Ca}^{2+}$ signalling

$2-\mathrm{APB}$ is known to modulate a variety of plasma membrane channels that are expressed in platelets, including Orai1, TRPC6 and $\mathrm{Ca}^{2+}$-activated $\mathrm{K}^{+}$channels ${ }^{24-28}$. To determine whether any of these targets were involved in the inhibitor effect of 2-APB, Cal-520-loaded platelets were used to monitor the effect of A23187 and 2APB on cytosolic $\mathrm{Ca}^{2+}$ signalling. A23187 induced a rapid increase in fluorescence (Fig. 3a). Surprisingly, 2-APB inhibited this increase in fluorescence in a concentrationdependent manner (Fig. 3a, b). Although DPBA also inhibited the Cal-520 fluorescence in a concentrationdependent manner, DP3A had no effect (Fig. 3a, b). This is important as DP3A inhibited $\mathrm{AnV}^{+} \mathrm{EV}$ release, suggesting that there is no direct link between the inhibition of Cal-520 fluorescence and inhibition of $\mathrm{AnV}^{+} \mathrm{EV}$ release. The analogues that did not affect $\mathrm{AnV}^{+} \mathrm{EV}$ release (PBA, DPTHF, DPHD, and DMBA) had no effect on the A23187-induced increase in fluorescence (Fig. 3c).

Inhibition of Orai1 with Synta-66 $(10 \mu \mathrm{M})$, TRPC6 with SAR-7334 $(1 \mu \mathrm{M})$ or the $\mathrm{Na}^{+}-\mathrm{Ca}^{2+}$ exchanger (NCX) with SN-6 $(50 \mu \mathrm{M})$, singly or combined, had no effect on the A23187-induced increase in Cal-520 fluorescence, $\mathrm{AnV}^{+} \mathrm{EV}$ release or platelet AnV binding (Fig. 3d-f).

Since it was surprising that 2-APB affected A23187induced cytosolic $\mathrm{Ca}^{2+}$ signalling, we investigated whether the effect on Cal-520 fluorescence might be an artefact owing to a change in cytosolic $\mathrm{pH}$. Addition of $\mathrm{NH}_{4} \mathrm{Cl}$ to unstimulated Cal-520-loaded platelets, which is expected to result in rapid intracellular alkalinisation, gave a rapid increase in fluorescence. Similarly, addition of extracellular $\mathrm{NaOH}$ also increased the fluorescence. Extracellular $\mathrm{HCl}$ had little effect on the Cal-520 fluorescence in unstimulated platelets (Fig. 4a). Although A23187 rapidly increased Cal-520 fluorescence to a plateau, which is as expected as the dye is likely to be saturated with $\mathrm{Ca}^{2+}$ under these conditions, this fluorescence could be further increased by extracellular $\mathrm{NH}_{4} \mathrm{Cl}$ or $\mathrm{NaOH}$. It could also be decreased by extracellular $\mathrm{HCl}$, suggesting that $\mathrm{H}^{+}$ enters stimulated platelets (Fig. 4b). Together, these data show that Cal-520 fluorescence is highly sensitive to $\mathrm{pH}$.
To determine whether 2-APB affects cytosolic $\mathrm{pH}$, platelets were loaded with the $\mathrm{pH}$-sensitive fluorescent dye, BCECF. $\mathrm{NH}_{4} \mathrm{Cl}$ rapidly increased BCECF fluorescence, indicating cytosolic alkalinisation, which slowly reversed (Fig. 4c). HCl rapidly decreased BCECF fluorescence, indicating cytosolic acidification (Fig. 4c). However, neither 2-APB, nor any of the analogues tested, significantly affected BCECF fluorescence (Fig. 4c, d). This suggests that 2-APB does not affect cytosolic $\mathrm{pH}$. Moreover, the effect of 2-APB and DPBA on Cal-520 fluorescence is not an artefact of decreased cytosolic $\mathrm{pH}$. Furthermore, altering cytosolic $\mathrm{pH}$ with $\mathrm{NH}_{4} \mathrm{Cl}$ or $\mathrm{HCl}$ did not significantly affect A23187-induced $\mathrm{AnV}^{+} \mathrm{EV}$ release or platelet AnV binding (Fig. 4e, f). These data indicate that a change in cytosolic $\mathrm{pH}$ does not account for the inhibitory effect of 2-APB.

2 -APB is also reported to block $\mathrm{Ca}^{2+}$-activated $\mathrm{K}^{+}$ channels $^{28}$, which may be required for PS-exposing EV release $^{29}$. However, neither quinine $(300 \mu \mathrm{M})$ nor tetraethylammonium (TEA; $30 \mathrm{mM}$ ), two non-selective blockers of $\mathrm{Ca}^{2+}$-activated $\mathrm{K}^{+}$channels, inhibited $\mathrm{AnV}^{+} \mathrm{EV}$ release or platelet AnV binding (Supplementary Fig. 3).

\section{2-APB, DBPA and DP3A inhibit $\mathrm{AnV}^{+}$EV release from permeabilised platelets}

To determine whether the effect of 2-APB was due to directly interfering with the action of A23187, a different means of inducing $\mathrm{AnV}^{+} \mathrm{EV}$ release was used. Platelets were permeabilised with the pore-forming toxin, streptolysin-O (SL-O). The efficiency of permeabilisation was tested by loading platelets with calcein. SL-O treatment led to loss of intracellular calcein. (Fig. 5a). Permeabilisation did not require extracellular $\mathrm{Ca}^{2+}$, though was enhanced by it (Fig. $5 \mathrm{~b}$ ). $\mathrm{CaCl}_{2}$ addition to permeabilised platelets triggered $\mathrm{AnV}^{+} \mathrm{EV}$ release (Fig. 5c). $\mathrm{AnV}^{+} \mathrm{EV}$ release was inhibited by the calpeptin $(140 \mu \mathrm{M})$, indicating that calpain is required (Fig. 5c), which is similar to A23187-induced $\mathrm{AnV}^{+}$EV release (Fig. 5d and ref. ${ }^{22}$ ).

$\mathrm{AnV}^{+} \mathrm{EV}$ release from permeabilised platelets was inhibited by 2-APB, DPBA and DP3A, but not the other analogues (Fig. 5c, d). This is the same pattern of action as seen with A23187-induced $\mathrm{AnV}^{+}$EV release. None of the 

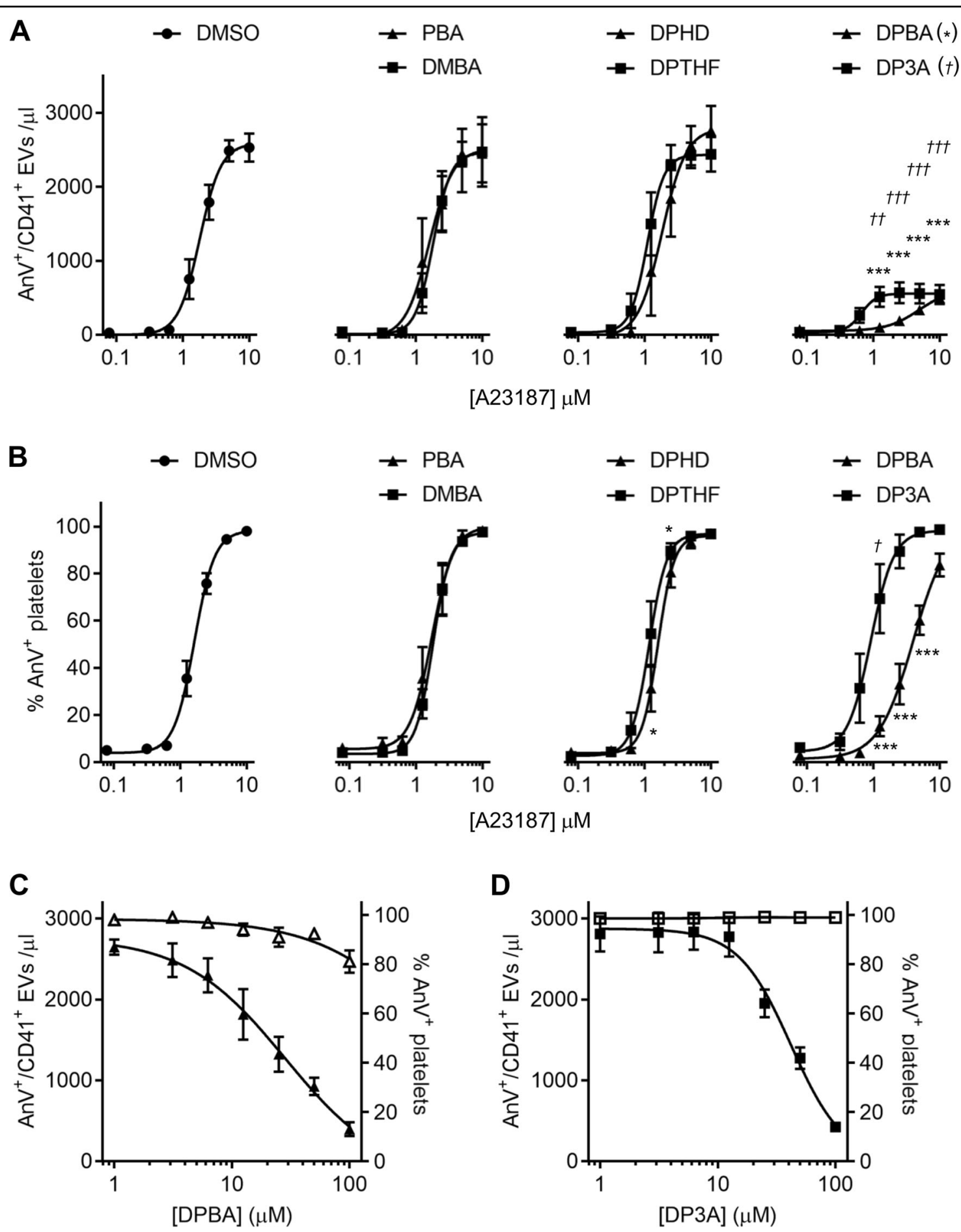

Fig. 2 Structural analogues of 2-APB show differing effects on $\mathbf{A n V}^{+}$EV release. $\mathbf{a}$, $\mathbf{b}$ Platelets were treated with the indicated analogue $(100 \mu \mathrm{M} ; 30 \mathrm{~min})$, or DMSO as control, then stimulated with a concentration range of A23187. AnV ${ }^{+}$EV release and platelet AnV binding were determined as described in Fig. 1. Concentration-response curves were fitted with a four-parameter logistic equation. Data were further analysed by two-way RM-ANOVA with Sidak's post-test. ${ }^{*} p<0.05 ;{ }^{* *} p<0.01 ;{ }^{* * *} p<0.001$ for comparison with DMSO-treated platelets. For clarity, in the righthand panel, statistical significance for DP3A is marked with $+\left({ }^{\dagger+} p<0.01 ;{ }^{+\dagger} p<0.001\right)$ but also indicates comparison with DMSO-treated platelets. $\mathbf{c}$, $\mathbf{d}$ Platelets were treated with a concentration range of DPBA (c) or DP3A (d) prior to stimulation with A23187 (10 $\mu \mathrm{M})$. 

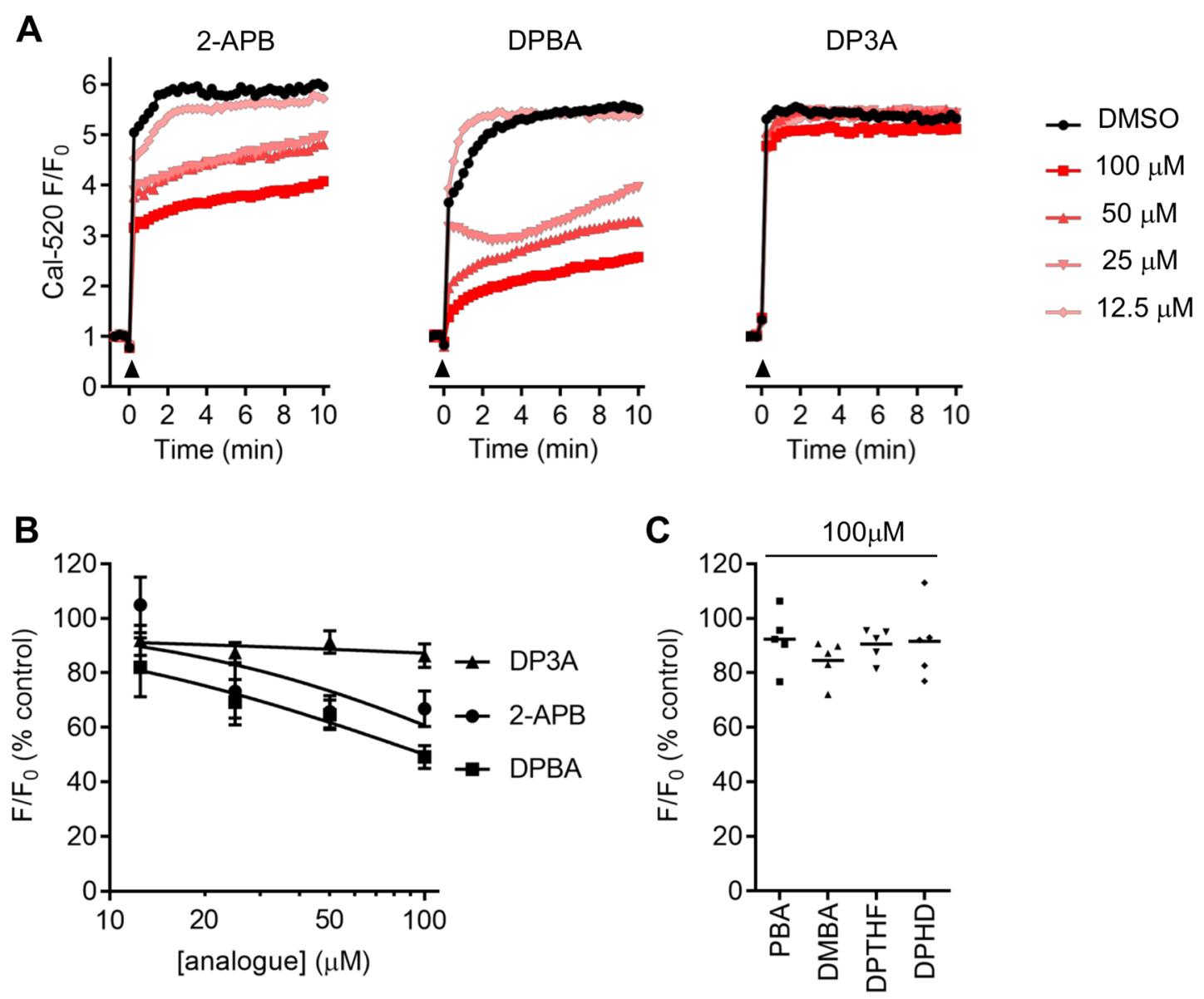

D

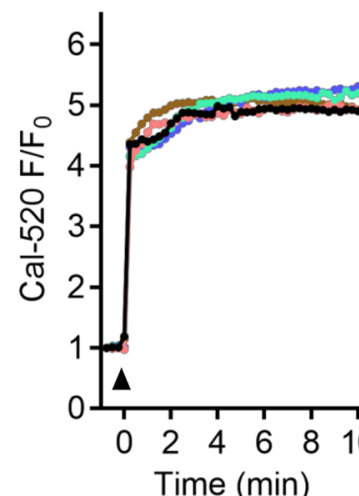

$\rightarrow$ DMSO

- SN-6

- SAR 7334
E

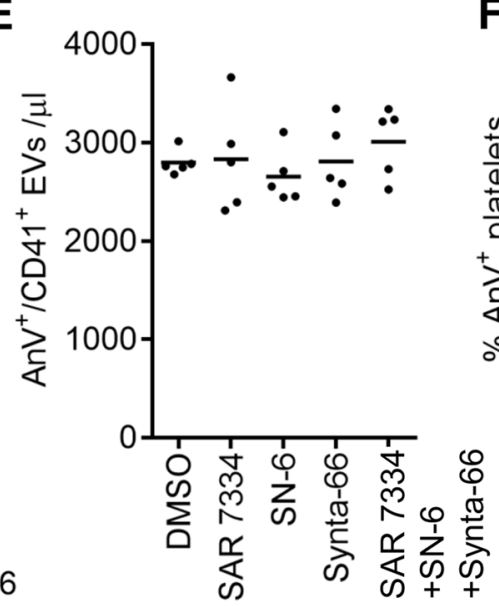

F

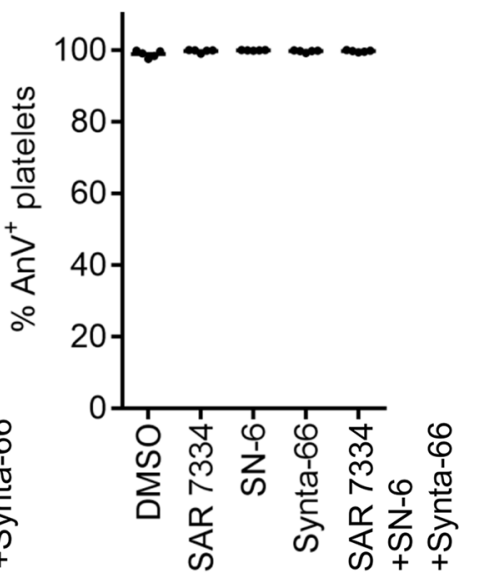
SAR 7334

$\rightarrow+\mathrm{SN}-6$

+Synta-66

Fig. 3 2-APB, DPBA and DP3A reduce Cal-520 fluorescence. a Representative traces of Cal-520-loaded platelets treated as indicated then stimulated with A23187 (10 $\mu \mathrm{M}$; black arrowhead), expressed as Cal-520 fluorescence normalised to the fluorescence prior to stimulation $\left(F / F_{0}\right)$. b Mean ( \pm s.e.m.; $n=5$ ) of maximum $F / F_{0}$ in each condition, as a percentage of DMSO-treated platelets. c Maximum $F / F_{0}$ for the inactive analogues

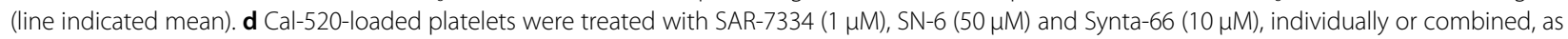
indicated, before stimulation with A23187 (10 $\mu \mathrm{M}$; black arrowhead). The traces are representative of five independent experiments. e, $\mathbf{f}$ AnV ${ }^{+}$EV release and platelet AnV binding in samples treated with these inhibitors. No statistically significant difference was observed compared to platelets treated with the solvent, DMSO (in each, one-way RM-ANOVA; $n=5$ ). 

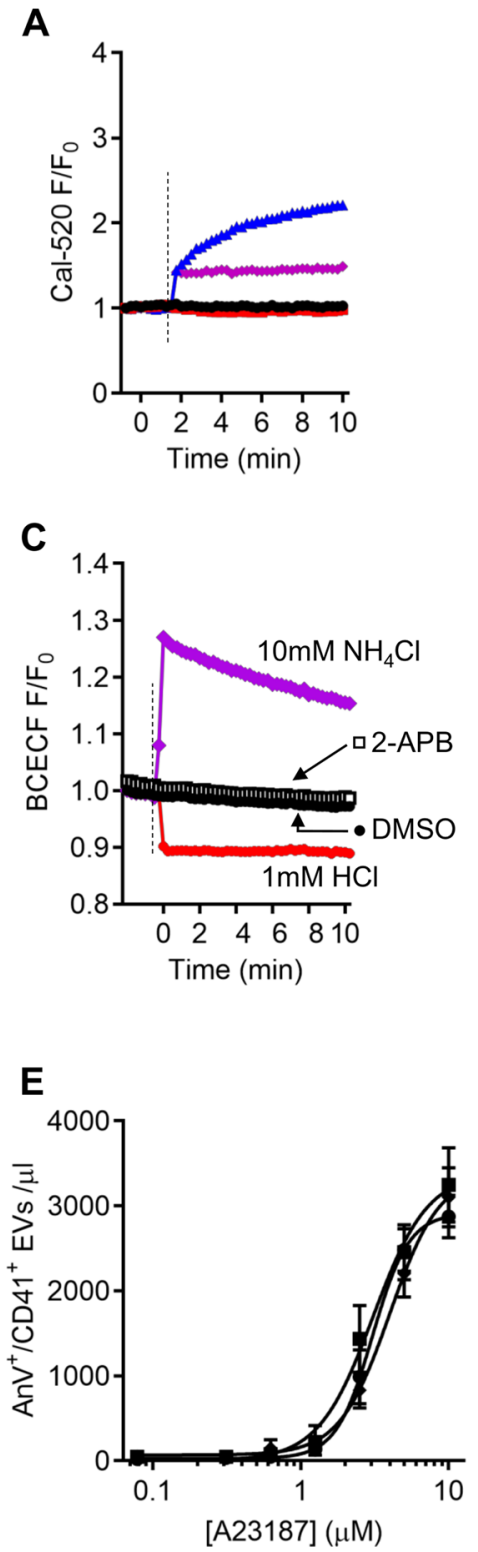

B
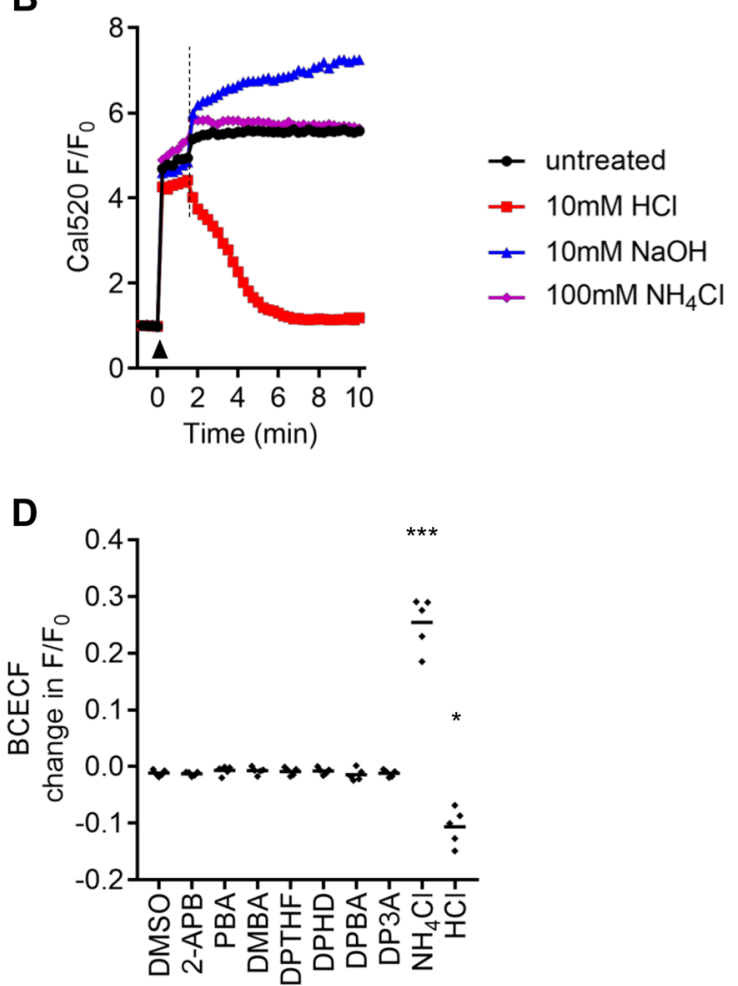

$\mathbf{F}$

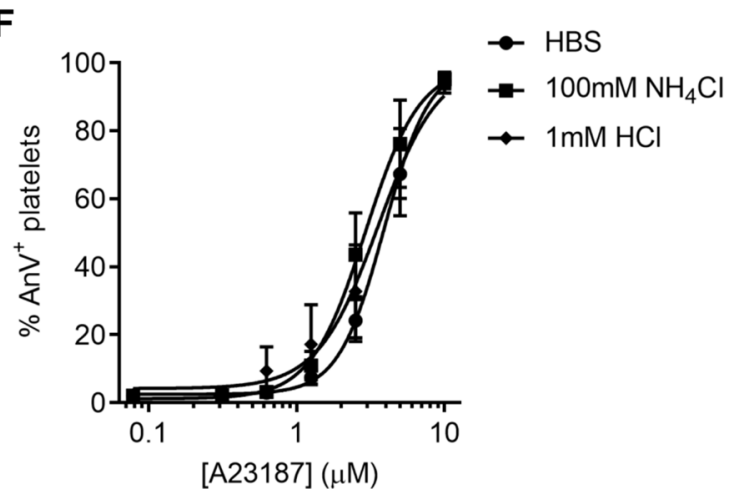

Fig. 4 2-APB does not inhibit $\mathbf{A n V}^{+}$EV release by altering cytosolic pH. a Cal-520-loaded platelets were treated with $\mathrm{HCl}(10 \mathrm{mM}), \mathrm{NaOH}$ $(10 \mathrm{mM})$ or $\mathrm{NH}_{4} \mathrm{Cl}(100 \mathrm{mM})$ at the point indicated by the dashed line. b Cal-520-loaded platelets were stimulated with A23187 (10 $\mu \mathrm{M}$; black arrowhead) followed by $\mathrm{HCl}, \mathrm{NaOH}$ or $\mathrm{NH}_{4} \mathrm{Cl}$ (dashed line). The traces are representative of five independent experiments. c Platelets were loaded with the pH-sensitive dye, BCECF. $\mathrm{HCl}, \mathrm{NH}_{4} \mathrm{Cl}, 2$ - $\mathrm{APB}$ or its vehicle, DMSO, were added at the dashed line. $\mathbf{d}$ Quantification of the change in $\mathrm{BCECF}$ fluorescence within $1 \mathrm{~min}$ of addition of the compounds indicated. The line shows the mean. Data with each treatment were compared to DMSOtreated platelets by one-way RM-ANOVA, with Dunnett's post-test. ${ }^{*} p<0.05$; ${ }^{* *} p<0.001$. e, f Platelets were treated with $\mathrm{NH}_{4} \mathrm{Cl}$ or $\mathrm{HCl}$ prior to stimulation with A23187. Data are mean \pm s.e.m. $(n=5)$. No statistically significant differences were observed compared to platelets treated with the solvent, HBS (two-way RM-ANOVA).

active drugs inhibited calcein loss from SL-O-permeabilised platelets (Fig. 5e)

\section{2-APB and DPBA partially inhibits calpain activation, but DP3A does not}

Calpain is required for $\mathrm{AnV}^{+} \mathrm{EV}$ release following A23187 or SL-O treatment. Platelet stimulation with
A23187 resulted in cleavage of the calpain substrates, talin, ATP8A1, PTP1C and caspase-3, which was prevented by pre-treatment with calpeptin (Fig. 6a). For example, A23187 reduced the presence of the highest talin band to $8.5 \pm 3.4 \%$ of unstimulated (DMSO-treated) samples $(n=5)$, whereas in the presence of calpeptin, this band was not significantly changed to $96.7 \pm 6.1 \%$ of 

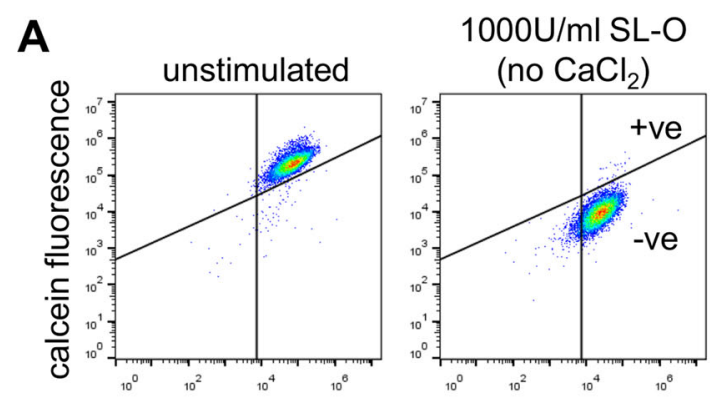

FSC
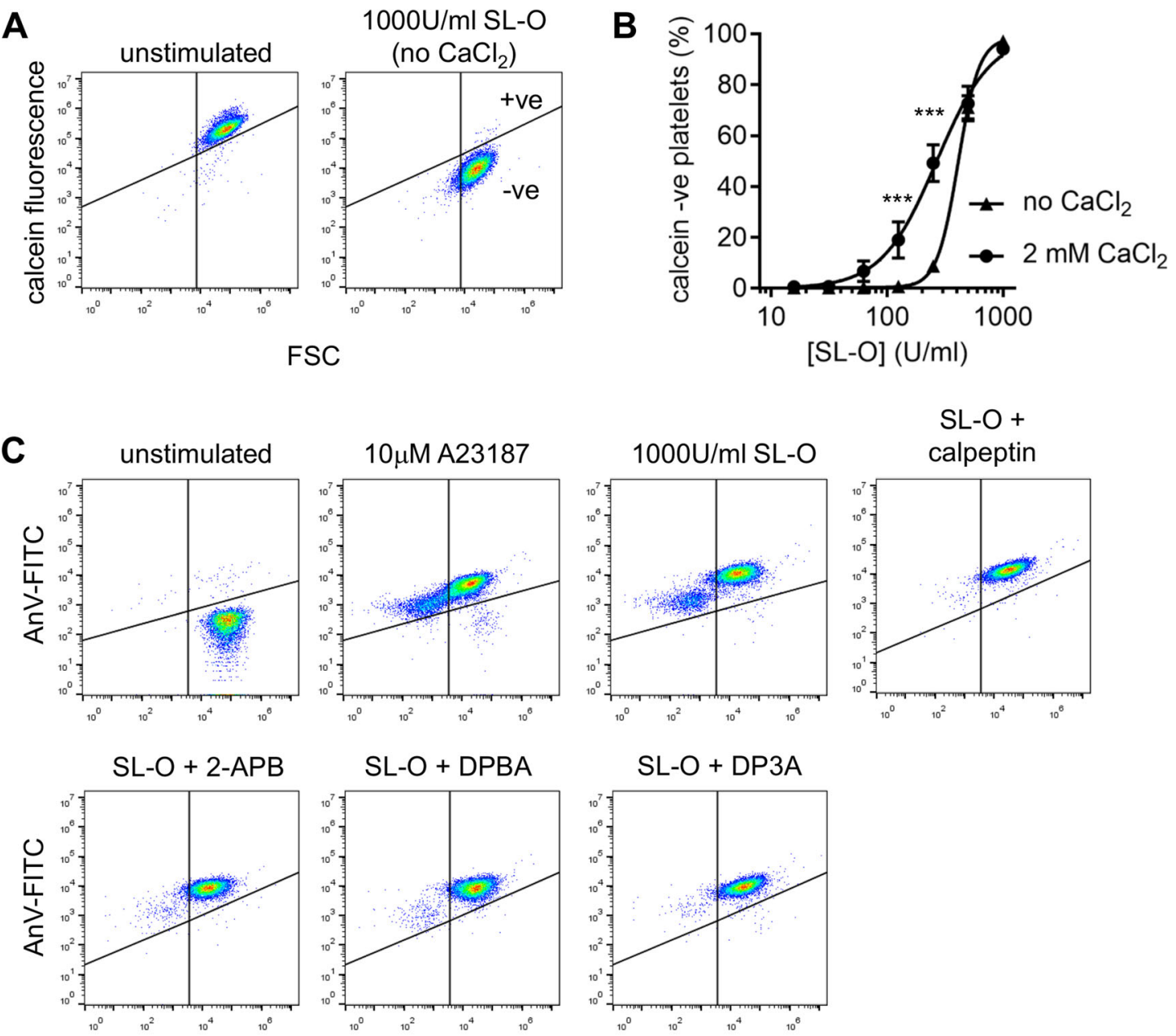

FSC

D

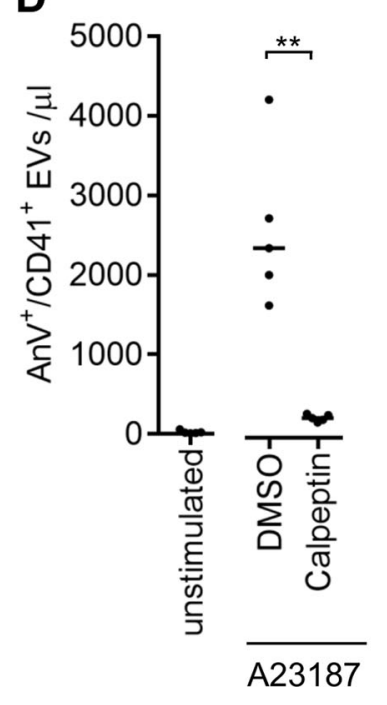

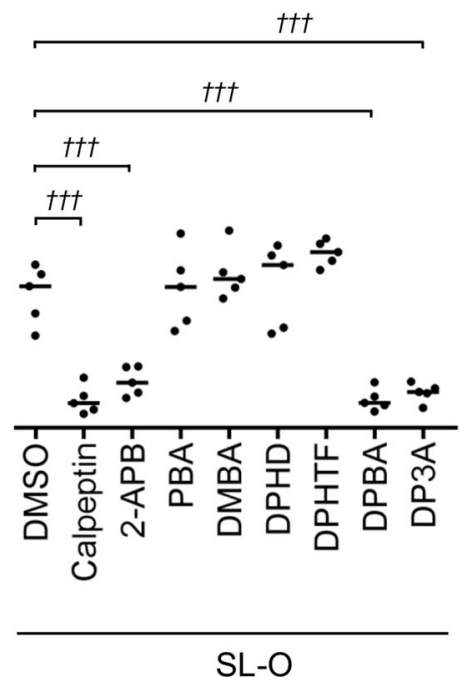

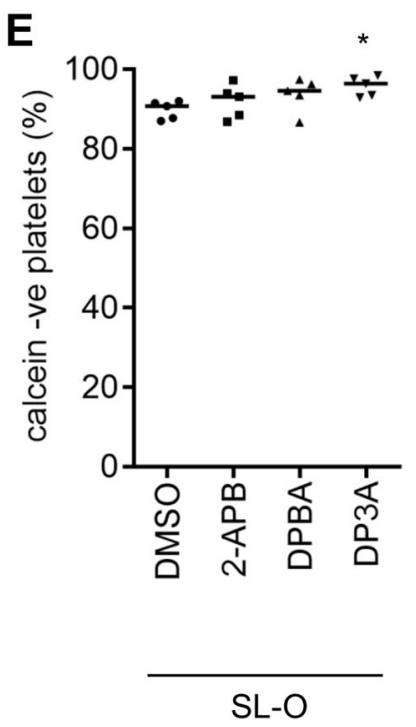

Fig. 5 (See legend on next page.) 
(see figure on previous page)

Fig. 5 2-APB, DPBA and DP3A inhibit streptolysin-O-induced $\mathrm{AnV}^{+}$release. $\mathbf{a}$, b Platelets were loaded with calcein then treated with reduced streptolysin-O (SL-O) in the presence or absence of extracellular $\mathrm{CaCl}_{2}$. Representative density plots are shown in (a). Unstimulated platelets have high calcein fluorescence. SL-O treated platelets have lost calcein. b Mean \pm s.e.m. $(n=5)$ of percentage of platelets that lost calcein fluorescence at various SL-O concentrations in the presence or absence of extracellular $\mathrm{CaCl}_{2}$. SL-O was more effective in the presence of extracellular $\mathrm{CaCl}_{2}{ }^{* * *} p<$ 0.001; two-way RM-ANOVA with Sidak's post-test). c Platelets (without calcein) were treated with $\mathrm{SL}-\mathrm{O}$ then $\mathrm{CaCl}_{2}$ added. AnV ${ }^{+} \mathrm{EVs}_{\mathrm{s}}$ were released in a similar pattern to A23187-stimulated platelets. Where indicated, platelets had been pre-treated with calpeptin $(140 \mu \mathrm{M} ; 30 \mathrm{~min})$ or 2-APB, DPBA or DP3A (all $100 \mu \mathrm{M}$ ). Density plots are representative of five independent experiments. $\mathbf{d}$ AnV ${ }^{+}$EV release from platelets stimulated with A23187 or $\mathrm{SL}-\mathrm{O}$, pre-treated as indicated. ${ }^{* *} p<0.01$ (calpeptin vs. DMSO, A23187 stimulated; paired $t$ test); ${ }^{{ }^{\dagger \dagger}} p<0.001$ (treatments indicated vs. DMSO, SL-Opermeabilised platelets; one-way RM-ANOVA; other treatments were not significantly different $p>0.05$ ). e Calcein-loaded platelets were treated with 2-APB, DPBA or DP3A, then permeabilised with SL-O. The percentage of platelets negative for calcein fluorescence in five independent experiments is shown. ${ }^{*} p<0.05$ compared to DMSO (one-way RM-ANOVA with Dunnett's post-test).

unstimulated, calpeptin-treated samples. Likewise, stimulation with A23187 led to the presence of a lower band (approximately $30 \mathrm{kDa}$ ) detected by the anti-caspase- 3 antibody, consistent with previous reports ${ }^{30}$. However, in calpeptin-treated samples, stimulation of A23187 led to a barely detectable band, only $4.4 \pm 1.0 \%(n=5)$ of the band in DMSO-treated, A23187-stimulated samples. 2-APB $(100 \mu \mathrm{M})$ also partially inhibited the cleavage of these substrates. In 2-APB-treated samples, A23187 reduced the highest band to $57.0 \pm 11.0 \%$ compared to DMSO, unstimulated samples $(p<0.05$; also $p<0.05$ compared to DMSO, A23187-stimulated; one-way ANOVA with Tukey's multiple comparisons test). Similarly, in 2-APBtreated samples, the lower caspase- 3 band was only $40.8 \pm$ $8.3 \%$ of that in DMSO-treated, A23187-stimulated samples $(p<0.05 ; n=5)$. The 2 -APB analogues were then tested for their ability to prevent the cleavage of two of these substrates, talin and caspase-3. Of the 2-APB analogues tested, DPBA also partially inhibited the cleavage of talin and capase-3, but DP3A, PBA, DMBA, DPHD and DPTHF had no effect (Fig. 6b).

\section{2-APB also inhibits $\mathrm{AnV}^{+} \mathrm{EV}$ release from endothelial cells}

To determine whether the inhibitory effect of 2-APB was restricted to platelets, human umbilical vein endothelial cells (HUVECs) were stimulated with A23187 $(10 \mu \mathrm{M})$, resulting in $\mathrm{AnV}^{+}$binding to HUVECs and release of $\mathrm{AnV}^{+} \mathrm{EVs}$ (Fig. 7a). $\mathrm{AnV}^{+} \mathrm{EV}$ release was prevented by calpeptin, indicating that it is dependent on calpain activity (Fig. 7a). Pre-treatment with 2-APB $(100 \mu \mathrm{M})$ inhibited the release of $\mathrm{AnV}^{+} \mathrm{EVs}$ but not HUVEC AnV binding. The $\mathrm{pIC}_{50}$ for inhibition of $\mathrm{AnV}^{+}$ EV release was $5.88 \pm 0.21$ (Fig. $7 \mathrm{~d} ; n=5$ ), showing that 2 APB is more potent in HUVEC than platelets. The same range of 2-APB analogues was then tested (Fig. 7b, c). PBA unexpectedly increased $\mathrm{AnV}^{+} \mathrm{EV}$ release. DMBA caused a significant loss of HUVEC viability, which may explain the increase in $\mathrm{AnV}^{+} \mathrm{EV}$ release that it caused, and was not used further (Fig. 7c). $\mathrm{AnV}^{+} \mathrm{EV}$ release was inhibited by DPBA (Fig. 7f; pIC $50=6.03 \pm 0.11 ; n=5$ ) and DP3A (Fig. 7e; $\mathrm{pIC}_{50}=5.52 \pm 0.32$ ), as in platelets.
However, in contrast to platelets, DPTHF also inhibited $\mathrm{AnV}^{+}$EV release (Fig. 7g; pIC $50=5.68 \pm 0.20$ ). DPHD also inhibited $\mathrm{AnV}^{+} \mathrm{EV}$ release, but only at $100 \mu \mathrm{M}$. These data suggest that the pharmacology of $\mathrm{AnV}^{+} \mathrm{EV}$ release from HUVEC is similar, but not identical, to that of platelets.

\section{Discussion}

PS-exposing EVs released by activated platelets are prothrombotic and proinflammatory ${ }^{11}$, and so inhibiting their release may be a beneficial therapeutic approach ${ }^{31}$. However, little is known about the mechanisms that lead to their release and there are few drugs that can selectively inhibit this process. In this study, we demonstrate that 2APB, a widely used $\mathrm{Ca}^{2+}$ channel modulator, inhibits PSexposing EV release. This is not through inhibition of the major pathways currently known to be required for release of PS-exposing EVs and is not through inhibition of currently known targets of 2-APB. Although 2-APB itself is insufficiently selective or potent to be a useful therapeutic inhibitor of EV release, our results suggest the existence of a potential target. Future identification of this target may aid the development of therapeutic drugs that prevent the release of PS-exposing EVs.

Although little is known about the mechanisms of PSexposing EV release from activated platelets, it requires a high, sustained increase in $\left[\mathrm{Ca}^{2+}\right]_{\mathrm{cyt}}{ }^{32-34}$. This can be triggered by a $\mathrm{Ca}^{2+}$ ionophore. Although this is not a physiological stimulation, it should bypass intracellular $\mathrm{Ca}^{2+}$ release channels, such as $\mathrm{IP}_{3} \mathrm{Rs}$, and plasma membrane $\mathrm{Ca}^{2+}$ entry channels, such as Orail and TRPC6, major targets for 2-APB in platelets ${ }^{24-26,35,36}$. Indeed, this is why we were not expecting 2-APB to inhibit A23187induced release of $\mathrm{AnV}^{+}$EVs. In contrast, if physiological activators were used, such as thrombin plus cross-linked collagen-related peptide, the increase in $\left[\mathrm{Ca}^{2+}\right]_{\text {cyt }}$ would require these $\mathrm{Ca}^{2+}$ channels ${ }^{37,38}$. The multiple effects of 2 -APB mean that it would not itself be a suitable therapeutic, although understanding its mechanism of action may help us to understand the mechanisms that underlie the release of PS-exposing EVs from platelets. 

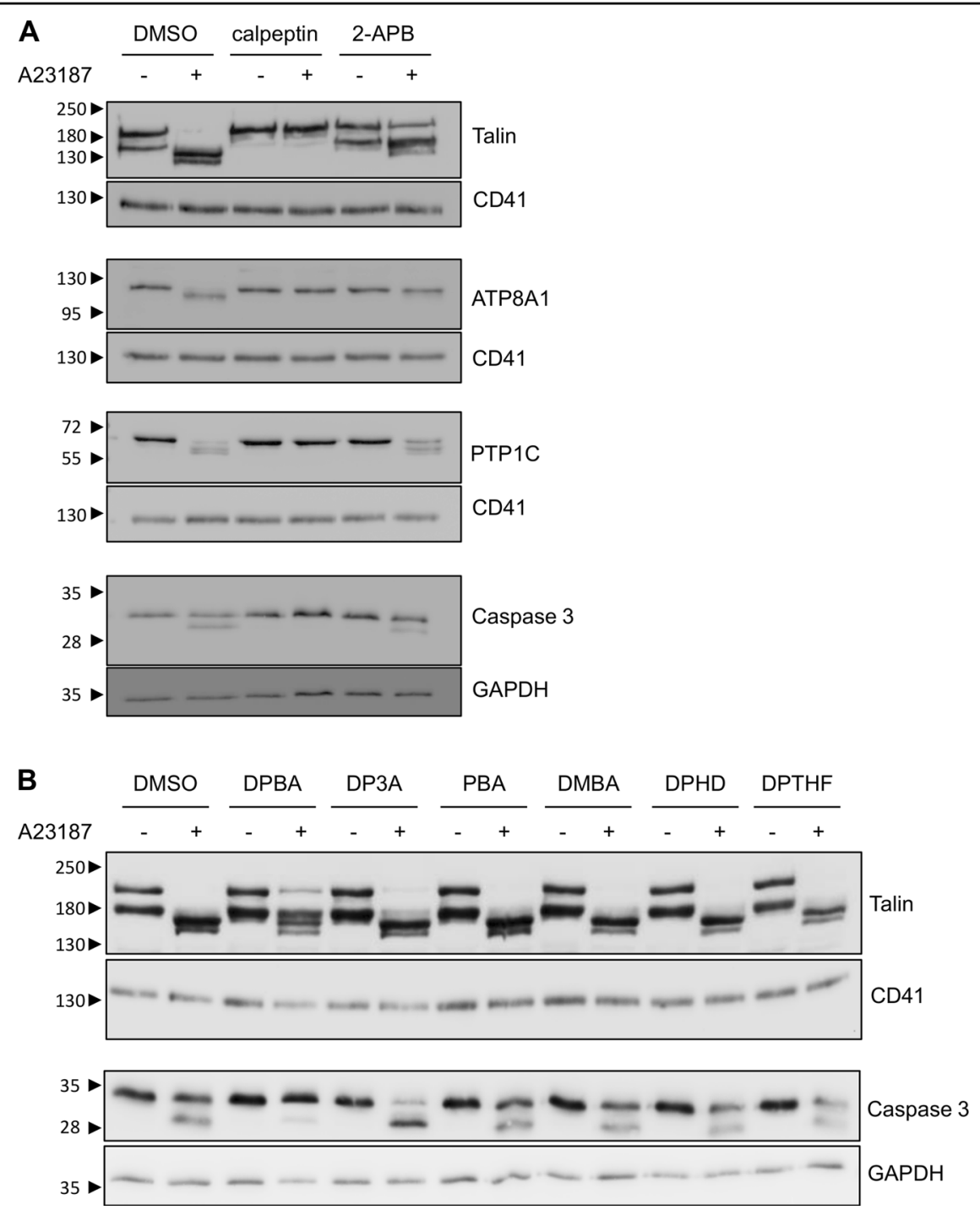

Fig. 6 Calpain activity is inhibited by 2-APB and DPBA, but not DP3A. a Platelets were treated with the calpain inhibitor, calpeptin (140 $\mu$ M), $2-$ APB $(100 \mu \mathrm{M})$ or the vehicle (DMSO) prior to stimulation with A23187 as indicated, then lysed. Proteins were separated by SDS-PAGE. Talin, ATP8A1, PTP1C and caspase-3 were detected with specific antibodies. The membranes were then stripped and re-probed with anti-CD41-antibody or antiGADPH antibody, as indicated. A23187-triggered protein cleavage was inhibited by calpeptin and partially inhibited by 2-APB. The blots are representative of five independent experiments. b Platelets were treated with the indicated analogues prior to stimulation with A23187. The blots are representative of five independent experiments.

Increased $\left[\mathrm{Ca}^{2+}\right]_{\text {cyt }}$ activates the $\mathrm{Ca}^{2+}$-dependent scramblase, TMEM16F, leading to PS exposure, and activation of the protease, calpain. Both events are required for PS-exposing EV release ${ }^{34}$. It is not likely that 2-APB directly inhibits TMEM16F, as platelet AnV binding was largely unaffected under conditions where $\mathrm{AnV}^{+}$EV release was completely inhibited. Moreover, although a small inhibition of platelet AnV binding was observed when 2-APB was present, this was fully reversed by washing the platelets, whereas the inhibition of $\mathrm{AnV}^{+}$ EV release was not so readily reversed.
Since 2-APB has many identified targets, we characterised a small set of related molecules that might have differing pharmacology. Two analogues, DPBA and DP3A, retained efficacy with similar potency to 2-APB. The other analogues showed no inhibitory action at $100 \mu \mathrm{M}$ in platelets. These observations indicated that neither the secondary amine, nor the boron atom, are absolutely required for the inhibitory action. They also suggest that the effect of 2-APB is not through inhibition of store-operated $\mathrm{Ca}^{2+}$ entry (SOCE), since DPTHF and DMBA (and to a small extent DPHD) have been shown to 


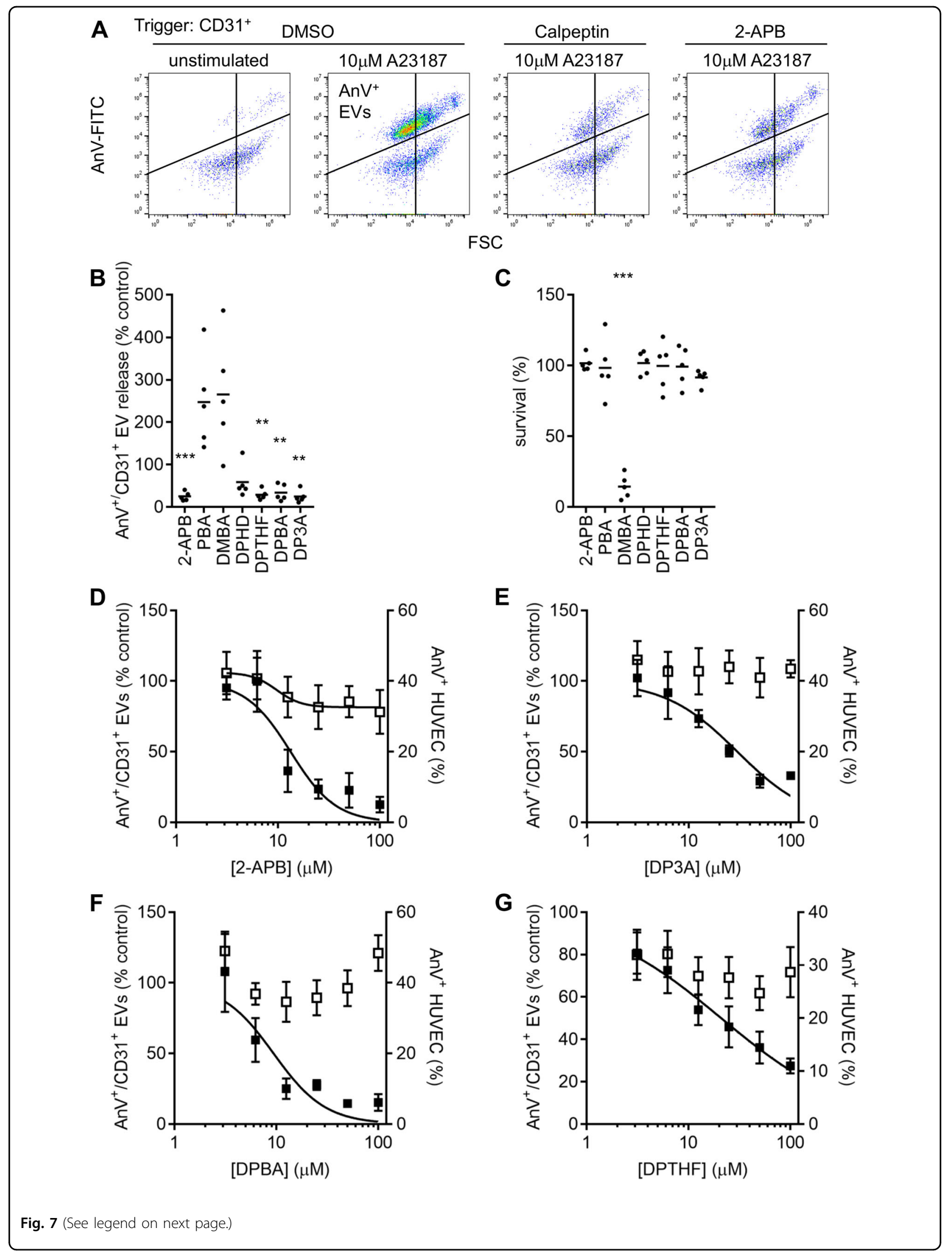


(see figure on previous page)

Fig. 7 2-APB, DPBA and DP3A inhibit AnV ${ }^{+}$EV release from HUVEC. a HUVEC were stimulated with A23187 (10 $\mu$ M) as described in "Methods". The panels show acquired events in the medium, stained with anti-CD31-APC and annexin V-FITC, using APC fluorescence to trigger acquisition. For comparison, some samples were pre-treated with calpeptin or 2-APB prior to stimulation. The density plots are representative of five independent experiments. $\mathbf{b}$ HUVEC were pretreated with 2-APB or one of its analogues then stimulated with A23187. AnV $V^{+}$EV release into the medium in expressed as a percentage of $\mathrm{AnV}^{+} \mathrm{EV}$ released in matched samples treated with the vehicle, DMSO, prior to A23187. ${ }^{* *} p<0.01,{ }^{* * *} p<0.001 \mathrm{vs}$. DMSO; one-way ANOVA with Holm-Sidak's post-test $(n=5)$. (c) HUVEC survival following treatment with 2-APB or its analogues was determined using a luminescence assay. ${ }^{* *} p<0.001$. $\mathbf{~ d - g}$ Platelets were treated with a concentration range of the indicated analogue prior to stimulation with prior to stimulation with $\mathrm{A} 23187(10 \mu \mathrm{M})$. $\mathrm{AnV}^{+} \mathrm{EV}$ release is expressed as a percentage of matched samples treated with the vehicle, DMSO, prior to A23187 $(n=5)$.

inhibit $\mathrm{SOCE}^{39}$ but had no effect on $\mathrm{AnV}^{+} \mathrm{EV}$ release. Interestingly, the pattern of active and inactive analogues was similar to that reported for interleukin (Il)- $1 \beta$ release from mouse macrophages ${ }^{40}$ (see Supplementary Table 1). In that study the effect was attributed to inhibition of the NLRP3 inflammasome. However, since Il-1 $\beta$ may be released in $\mathrm{EVs}^{41}$, inhibition of $\mathrm{EV}$ release could contribute to the inhibition of Il-1 $\beta$ release.

Moreover, these analogues provided a small toolkit to help explore the mechanism of action of 2-APB. $\mathrm{AnV}^{+} \mathrm{EV}$ release showed the same pattern of sensitivity to 2-APB analogues whether the process was triggered by $\mathrm{A} 23187$ in intact platelets or by $\mathrm{Ca}^{2+}$ addition to SL-O-permeabilised platelets. This supports the assumption that the two stimuli were triggering the same process. It also indicates that the lack of action of PBA, DMBA, DPHD and DPTHF was not due to insufficient plasma membrane permeability.

2-APB is unlikely to inhibit $\mathrm{AnV}^{+} \mathrm{EV}$ release through an action on $\mathrm{Ca}^{2+}$ channels. This is shown by several lines of evidence. The most direct evidence is the observation that 2-APB also inhibited $\mathrm{AnV}^{+} \mathrm{EV}$ release from SL-Opermeabilised platelets. SL-O permeabilisation allowed calcein to completely leak from the platelets, and has been previously used to introduce antibodies into platelets ${ }^{42}$. Extracellular $\mathrm{Ca}^{2+}$ rapidly triggered $\mathrm{AnV}^{+} \mathrm{EV}$ release from SL-O-permeabilised platelets in a calpaindependent manner. Together, these data indicate that there is direct continuity between the extracellular and intracellular environment. 2-APB did not prevent calcein leakage from SL-O-permeabilised platelets, indicating that it did not affect this continuity. Therefore, it is unlikely that 2-APB inhibits $\mathrm{AnV}^{+} \mathrm{EV}$ release by inhibiting $\mathrm{Ca}^{2+}$ flux between the extracellular and intracellular environment. Consistent with this conclusion, inhibition of Orail or TRPC6 (or NCX, which acts in reverse-mode downstream of TRPC ${ }^{37}$ ) did not affect $\mathrm{AnV}^{+} \mathrm{EV}$ release. Moreover, it is unlikely that other ion fluxes, such as $\mathrm{Na}^{+}, \mathrm{K}^{+}$or $\mathrm{Cl}^{-}$are required. For example, $\mathrm{Ca}^{2+}$-activated $\mathrm{K}^{+}$channels have previously been proposed to regulate platelet PS exposure and subsequent $\mathrm{AnV}^{+} \mathrm{EV}$ release ${ }^{29}$, and these channels may be inhibited by $2-\mathrm{APB}^{28}$. However, not only is it unlikely that $\mathrm{K}^{+}$flux plays an important role once the platelets have been permeabilised, but also two unrelated, non-selective blockers of $\mathrm{Ca}^{2+}$-activated $\mathrm{K}^{+}$channels had no effect on A23187-induced $\mathrm{AnV}^{+}$EV release. Therefore, it is likely that 2-APB has a target in $\mathrm{AnV}^{+} \mathrm{EV}$ release that is separate to plasma membrane ion channels.

At first sight, our observation that 2-APB inhibits the A23187-induced increase in Cal-520 fluorescence appears to lead to the opposite conclusion, as it suggests that 2APB inhibits the increase in $\left[\mathrm{Ca}^{2+}\right]_{\mathrm{cyt}}$. It is unclear whether this is a real inhibition of $\left[\mathrm{Ca}^{2+}\right]_{\mathrm{cyt}}$, or an artefactual effect on Cal-520 fluorescence. It is possible that 2-APB and DPBA affect the fluorescence spectrum of Cal-520 (in a manner that DP3A does not). We first considered whether 2-APB might induce cytosolic acidification, as it has been previously shown to rapidly acidify Jurkat $\mathrm{T}$ cells ${ }^{43}$, though this required action on (unidentified) membrane proteins and may be a cell-type specific effect. Many $\mathrm{Ca}^{2+}$-sensitive fluorescent dyes are acutely sensitive to $\mathrm{pH}$ and our data show that $\mathrm{Cal}-520$ is also sensitive to $\mathrm{pH}$. However, 2-APB did not affect intracellular $\mathrm{pH}$, assessed by BCECF fluorescence, suggesting that cytosolic acidification does not explain the reduced Cal-520 fluorescence. In addition, treatments that did alter intracellular $\mathrm{pH}$ (extracellular addition of $\mathrm{NH}_{4} \mathrm{Cl}$ or $\mathrm{HCl}$ ) did not affect $\mathrm{AnV}^{+} \mathrm{EV}$ release. This indicates that 2-APB does not inhibit $\mathrm{AnV}^{+} \mathrm{EV}$ release by altering intracellular $\mathrm{pH}$.

It is possible that 2-APB does inhibit the A23187induced increase in $\left[\mathrm{Ca}^{2+}\right]_{\mathrm{cyt}}$, rather than artefactually decrease Cal-520 fluorescence, though the mechanisms that could be involved are unclear. The ionophore action of A23187 itself could be directly inhibited, which could be tested by using alternative approaches to increase $\left[\mathrm{Ca}^{2+}\right]_{\text {cyt }}$ (e.g. ionomycin, thapsigargin). Our data indicate that the action of 2-APB on A23187-induced Cal-520 fluorescence does not involve inhibition of Orail or TRPC6, the major $\mathrm{Ca}^{2+}$ entry channels in platelets. One possibility is that platelets contain two intracellular $\mathrm{Ca}^{2+}$ stores, the dense tubular system and acidic $\mathrm{Ca}^{2+}$ stores, such as lysosomes ${ }^{44}$. The latter are relatively insensitive to $\mathrm{Ca}^{2+}$ ionophores ${ }^{45}$ and may require a 2 -APB-sensitive process for their depletion. Inhibition of $\left[\mathrm{Ca}^{2+}\right]_{\mathrm{cyt}}$ signalling could account for the weak inhibition of PS 
exposure and calpain activity by 2-APB and DPBA. Although PS exposure and calpain activity are required for PS-exposing EV release ${ }^{5,20,22,46}$, these small inhibitory effects cannot account for the inhibition of $\mathrm{AnV}^{+} \mathrm{EV}$ release by $2-\mathrm{APB}$, since the phosphorus-containing analogue, DP3A, had no effect on Cal-520 fluorescence, platelet AnV binding or calpain activity, yet inhibited $\mathrm{AnV}^{+} \mathrm{EV}$ release with similar potency. These data suggest that there is a further target of 2-APB, independent of $\left[\mathrm{Ca}^{2+}\right]_{\mathrm{cyt}}$, PS exposure and calpain activity, that is required for $\mathrm{AnV}^{+} \mathrm{EV}$ release (Fig. 8). DP3A is likely to inhibit the same target, without these other effects, and may be a better scaffold from which to develop a more selective inhibitor of EV release.

To determine whether the action of 2-APB was specific to $\mathrm{AnV}^{+} \mathrm{EV}$ release from platelets, we also examined $\mathrm{AnV}^{+}$EV release from HUVECs. Again, we used A23187 to directly increase $\left[\mathrm{Ca}^{2+}\right]_{\mathrm{cyt}}$, aiming to bypass any inhibition of $\mathrm{Ca}^{2+}$ channels by 2-APB. Endothelial cells release EVs during activation and apoptosis ${ }^{47}$. Whether the mechanisms underlying the release of EVs under these different conditions are the same in unclear. In our experimental setting, A23187 rapidly triggered AnV binding to HUVEC and release of $\mathrm{AnV}^{+}$EVs. The latter was blocked by 2-APB. However, although $\mathrm{AnV}^{+} \mathrm{EV}$ release from HUVEC was also inhibited by DPBA and DP3A, as in platelets, these drugs were more potent in HUVEC than in platelets. Moreover, $\mathrm{AnV}^{+}$EV release from HUVEC was also inhibited by DPTHF. This suggests that the target of 2-APB in HUVEC and platelets may be

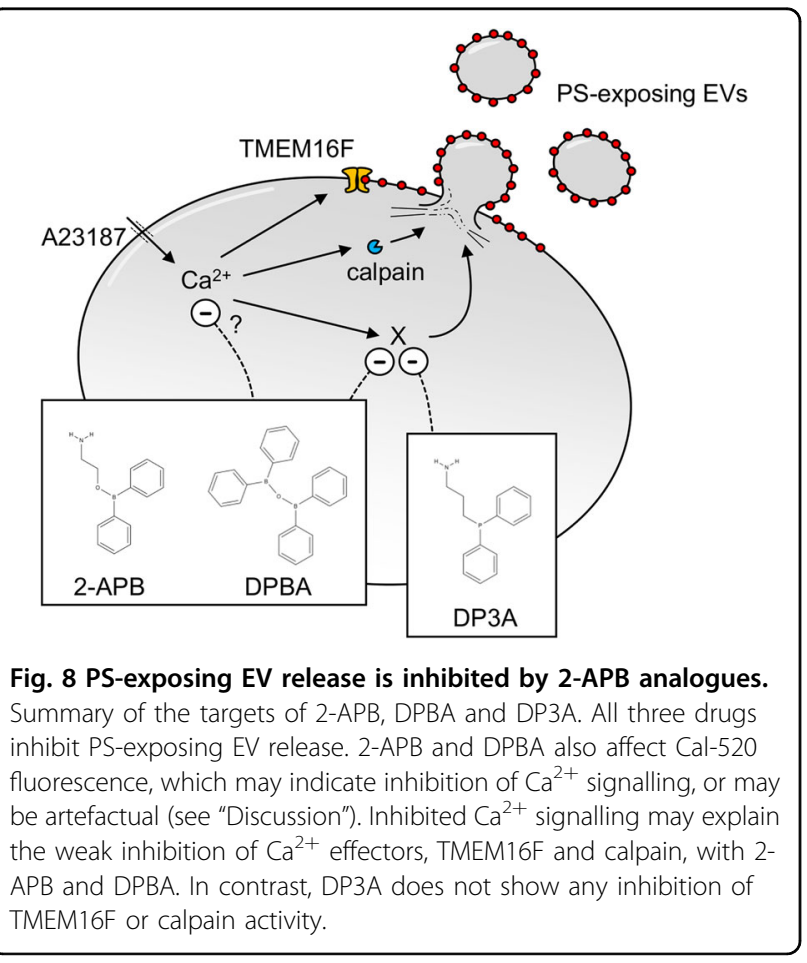

similar but are not identical. As a tentative speculation, it may involve different isoforms of a protein family. This difference opens the possibility of being able to selectively inhibit PS-exposing EV release from different cells.

\section{Conclusion}

PS-exposing EVs are released by stimulated platelets in $\mathrm{a} \mathrm{Ca}^{2+}$ /calpain-dependent manner. Although PS-exposing EVs are likely to contribute to a range of prothrombotic and proinflammatory diseases, there are few drugs available to block their release. 2-APB inhibits the release of $\mathrm{AnV}^{+}$EVs. Identifying the target of 2-APB, DPBA and DP3A may provide a new way to inhibit $\mathrm{AnV}^{+} \mathrm{EV}$ release from activated platelets and inhibit their contribution to thrombosis and inflammation.

\section{Methods}

\section{Washed platelet preparation}

Blood was drawn by venepuncture into sodium citrate $(3.2 \% \mathrm{v} / \mathrm{v})$ from healthy, drug-free volunteers, who had given written, informed consent in accordance with the Declaration of Helsinki. Use of human blood for these experiments was approved by the Human Biology Research Ethics Committee, University of Cambridge. Acid citrate dextrose (ACD; $85 \mathrm{mM}$ tri-sodium citrate, $71 \mathrm{mM}$ citric acid, $111 \mathrm{mM}$ D-glucose) was added (1:7 v/v) and platelet-rich plasma (PRP) separated by centrifugation $(200 g, 10 \mathrm{~min})$. Prostaglandin $\mathrm{E}_{1}$ $(100 \mathrm{nM})$ and apyrase (Grade VII; $0.02 \mathrm{U} / \mathrm{ml}$ ) were added to PRP to prevent platelet activation during preparation. Where required, platelets were incubated with either Cal-520acetoxymethyl (AM) or calcein-AM (both $1 \mu \mathrm{M} ; 10 \mathrm{~min}$ ). Platelets were pelleted from PRP by centrifugation $(600 g$, $10 \mathrm{~min}$ ) and resuspended in HEPES-buffered saline (in $\mathrm{mM}$ : 10 HEPES, $135 \mathrm{NaCl}, 3 \mathrm{KCl}, 0.34 \mathrm{NaH}_{2} \mathrm{PO}_{4}, 1 \mathrm{MgCl}_{2} \cdot 6 \mathrm{H}_{2} \mathrm{O}$, $\mathrm{pH}$ 7.4; supplemented with $0.9 \mathrm{mg} / \mathrm{ml} \mathrm{D-glucose)} \mathrm{at} 5 \times 10^{7}$ platelets $/ \mathrm{ml}$. Platelets were rested $\left(30^{\circ} \mathrm{C}, 30 \mathrm{~min}\right)$ prior to treatment with inhibitors or stimulation. $\mathrm{CaCl}_{2}(2 \mathrm{mM})$ was added immediately prior to simulation.

\section{Flow cytometry analysis}

Following stimulation, samples were stained with FITCconjugated annexin $\mathrm{V}$ (eBioscience, ThermoFisher, UK), to detect exposed PS, (FL1), unless otherwise indicated, and PECy7-conjugated anti-CD41 antibody (eBioscience, ThermoFisher, UK), to distinguish platelet-derived events. Samples were analysed using a BD Accuri C6 flow cytometer. PE-Cy7 fluorescence (FL3) was used to trigger event acquisition. PSpositive platelet-derived EVs were defined as $\mathrm{CD} 41^{+} /$annexin $\mathrm{V}^{+}$events that were smaller than $1 \mu \mathrm{m}$. The $1 \mu \mathrm{m}$ gate was set in forward scatter using $1 \mu \mathrm{m}$ silica beads ${ }^{22}$.

\section{Immunoblotting}

Platelet proteins were detected in platelet lysates by sodium dodecyl sulfate-polyacrylamide gel electrophoresis 
and immunoblotting, essentially as described previously ${ }^{22}$. The primary antibodies used were: anti-talin antibody (clone 8D4; T3287; from Sigma Aldrich, Poole, Dorset, UK) anti-PTP1C (610125; BD Biosciences); anti-ATP8A1 (21565-1-AP; Proteintech Europe, Manchester, UK); antiCD41 antibody (ab134131; from Abcam, Cambridge, UK); and anti-caspase3 (9662) and anti-GAPDH (2118; from Cell Signalling Technology; Davers MA, USA). The secondary antibodies used were horseradish peroxidase-conjugated anti-rabbit IgG (7074) or anti-mouse IgG (7076; both Cell Signalling Technology).

\section{Measurement of cytosolic $\mathrm{Ca}^{2+}$ concentration $\left(\left[\mathrm{Ca}^{2+}\right]_{\mathrm{cyt}}\right)$ and $\mathrm{pH}$}

Cal-520-loaded platelets were stimulated in black-walled microplates at $30^{\circ} \mathrm{C}$. Fluorescence (excitation: $492 \mathrm{~nm}$; emission: $520 \mathrm{~nm}$ ) was recorded using a FLUOStar OMEGA (BMG LabTech). BCECF-loaded platelets were stimulated in black-walled microplates at $30^{\circ} \mathrm{C}$. Fluorescence (excitation: $485 \mathrm{~nm}$; emission: $520 \mathrm{~nm}$ ).

\section{Measurement of endothelial EV release and PS exposure}

HUVEC (Promocell, c-12203), were cultured in endothelial cell growth medium (PromoCell; c-22010), supplemented with $35 \mu \mathrm{g} / \mathrm{mL}$ gentamycin and endothelial cell growth supplements (Promocell, c-39215) at 5\% $\mathrm{CO}_{2}$ and $37^{\circ} \mathrm{C}$. HUVEC were routinely tested for mycoplasma contamination. HUVEC were seeded into 24-well plates (at $1.5 \times 10^{5} /$ well) $24 \mathrm{~h}$ prior to drug treatment. HUVEC were washed to remove cell debris and constitutively released EVs then pre-treated for $30 \mathrm{~min}$ in endothelial cell growth media with the appropriate inhibitor. After this, media was replaced with HEPES-buffered saline supplemented with glucose $(0.9 \mathrm{mg} / \mathrm{ml}), \mathrm{CaCl}_{2}(2 \mathrm{mM}), \mathrm{A} 23187$ $(10 \mu \mathrm{M})$ and inhibitor for $10 \mathrm{~min}$. Following stimulation, both the EV-rich medium and trypsinized endothelial cells were collected and stained with APC-conjugated antiCD31 (eBioscience,ThermoFisher, UK). Samples were analysed using a BD Accuri C6 flow cytometer. APC fluorescence (FL4) was used to trigger event acquisition and identify endothelial (or endothelial-derived EV) events. to determine EV count and cellular PS exposure, respectively. PS-exposing endothelial-derived EVs were defined as $\mathrm{CD}^{2} 1^{+}$/annexin $\mathrm{V}^{+}$events that were smaller than $1 \mu \mathrm{m}$ in an analogous manner to platelet-derived EVs.

\section{Endothelial viability}

To determine inhibitor toxicity, HUVEC were seeded in a 96-well clear-bottom plate at $1.5 \times 10^{4}$ cells/well, $24 \mathrm{~h}$ prior to treatment. Endothelial cells were treated as described above. The cell viability assay (Promega, UK G7572) was conducted according to the manufacturer's instruction to evaluate the ATP content.

\section{Data presentation and statistical analysis}

Data are reported as mean \pm standard error of mean (SEM) from at least five independent platelet preparations. $N=5$ was chosen based on the mean and standard deviation of $\mathrm{AnV}^{+} \mathrm{EV}$ release in preliminary experiments, in order to detect a $50 \%$ reduction with $80 \%$ power at $p<$ 0.05. Data were compared using one-way or two-way repeat measures analysis of variance (RM-ANOVA), as appropriate, in GraphPad Prism v7. No samples were excluded from analysis. Concentration-response curves were fitted using a four-parameter logistical equation. This was also used to estimate $\mathrm{pIC}_{50}$ (i.e., $-\log \left(\mathrm{IC}_{50}\right)$ ).

\section{Source of materials}

All reagents, including the 2-APB analogues, were obtained from Sigma Aldrich (Poole, Dorset, UK) unless otherwise stated. A23187 was from Acros Organics (Fisher Scientific, UK). 2-APB was from Cayman Chemicals (Cambridge Bioscience, Cambridge, UK). SAR-7334 and SN-6 were from Tocris (Bristol, UK). Calcein and BCECF were from ThermoFisher Scientific. Cal-520 was from AAT Bioquest (Sunnyvale CA, USA).

\section{Acknowledgements}

J.E.D. was supported by British Heart Foundation Project Grant PG/16/45/32152 to M.T.H. Work in Dr. Harper's lab was also supported by Wellcome Trust Seed Award 200702/Z/16/Z and Royal Society Research Grant RG170381.

\section{Data availability}

The data are available from the corresponding author on reasonable request

Conflict of interest

The authors declare that they have no conflict of interest.

\section{Publisher's note}

Springer Nature remains neutral with regard to jurisdictional claims in published maps and institutional affiliations.

The online version of this article (https://doi.org/10.1038/s41420-020-0244-9) contains supplementary material, which is available to authorized users.

Received: 7 November 2019 Revised: 3 December 2019 Accepted: 10 December 2019

Published online: 02 March 2020

\footnotetext{
References

1. Jackson, S. P. Arterial thrombosis-insidious, unpredictable and deadly. Nat. Med. 17, 1423-1436 (2011).

2. Huber, K. et al. Antiplatelet and anticoagulation agents in acute coronary syndromes: what is the current status and what does the future hold? Am. Heart J. 168, 611-621 (2014).

3. Protasiewicz, M., Szymkiewicz, P., Kuliczkowski, W., Mysiak, A. \& Witkiewicz, W. Modern antiplatelet therapy—opportunities and risks. Adv. Clin. Exp. Med. 22 875-885 (2013).

4. Suzuki, J., Umeda, M., Sims, P. J. \& Nagata, S. Calcium-dependent phospholipid scrambling by TMEM16F. Nature 468, 834-838 (2010).

5. Fujii, T., Sakata, A., Nishimura, S., Eto, K. \& Nagata, S. TMEM16F is required for phosphatidylserine exposure and microparticle release in activated mouse platelets. Proc. Natl Acad. Sci. USA 112, 12800-12805 (2015).
} 
6. Millington-Burgess, S. L. \& Harper, M. T. Gene of the issue: ANO6 and Scott Syndrome. Platelets https://doi.org/10.1080/09537104.2019.1693039 (2019).

7. Lhermusier, T., Chap, H. \& Payrastre, B. Platelet membrane phospholipid asymmetry: from the characterization of a scramblase activity to the identification of an essential protein mutated in Scott syndrome. J. Thromb. Haemost. 9, 1883-1891 (2011).

8. van Geffen, J. P., Swieringa, F. \& Heemskerk, J. W. M. M. Platelets and coagulation in thrombus formation: aberrations in the Scott syndrome. Thromb. Res. 141, S12-S16 (2016)

9. Swieringa, F., Spronk, H. M. H., Heemskerk, J. W. M. \& van der Meijden, P. E. J. Integrating platelet and coagulation activation in fibrin clot formation. Res. Pract. Thromb. Haemost. 2, 450-460 (2018).

10. Heemskerk, J. W. M., Mattheij, N. J. A. \& Cosemans, J. M. E. M. Platelet-based coagulation: different populations, different functions. J. Thromb. Haemost. 11, 2-16 (2013).

11. Vajen, T., Mause, S. F. \& Koenen, R. R. Microvesicles from platelets: novel drivers of vascular inflammation. Thromb. Haemost. 114, 228-236 (2015).

12. Sun, C., Zhao, W. B., Chen, Y. \& Hu, H. Y. Higher plasma concentrations of platelet microparticles in patients with acute coronary syndrome: a systematic review and meta-analysis. Can. J. Cardiol. 32, 1325.e1-1325.e10 (2016).

13. Mavroudis, C. A. et al. Microparticles in acute coronary syndrome. Thromb. Res. 156, 109-116 (2017)

14. Preston, R. A. et al. Effects of severe hypertension on endothelial and platelet microparticles. Hypertension 41, 211-217 (2003).

15. Li, S. Y. et al. Cell-derived microparticles in patients with type 2 diabetes mellitus: a systematic review and meta-analysis. Cell. Physiol. Biochem. 39, 2439-2450 (2016).

16. Cortez-Espinosa, N. et al. Platelets and platelet-derived microvesicles as immune effectors in type 2 diabetes. Curr. Vasc. Pharmacol. 15, 207-217 (2017).

17. Wang, Z. T., Wang, Z. \& Hu, Y. W. Possible roles of platelet-derived microparticles in atherosclerosis. Atherosclerosis 248, 10-16 (2016).

18. Italiano, J. E., Mairuhu, A. T. A., Flaumenhaft, R. \& Flaumenhaft, R. Clinical relevance of microparticles from platelets and megakaryocytes. Curr. Opin. Hematol. 17, 578-584 (2010).

19. Sims, P. J., Wiedmer, T., Esmon, C. T., Weiss, H. J. \& Shattil, S. J. Assembly of the platelet prothrombinase complex is linked to vesiculation of the platelet plasma membrane. Studies in Scott syndrome: an isolated defect in platelet procoagulant activity. J. Biol. Chem. 264, 17049-17057 (1989).

20. Fox, J. E. B., Austin, C. D., Reynolds, C. C. \& Steffen, P. K. Evidence that agonistinduced activation of calpain causes the shedding of procoagulant-containing microvesicles from the membrane of aggregating platelets. J. Biol. Chem. $\mathbf{2 6 6}$ 13289-13295 (1991)

21. Bachelot-Loza, C. et al. Differential regulation of platelet aggregation and aminophospholipid exposure by calpain. Br. J. Haematol. 133, 419-426 (2006).

22. Wei, H., Malcor, J. \& Harper, M. Lipid rafts are essential for release of phosphatidylserine-exposing extracellular vesicles from platelets. Sci. Rep. 8 9987 (2018).

23. Finch, A. \& Gardner, P. J. Thermochemistry of phenylboronic acid, diphenylborinic acid, and their anhydrides. Trans. Faraday Soc. 62, 3314 (1966).

24. Dobrydneva, Y. \& Blackmore, P. 2-Aminoethoxydiphenyl borate directly inhibits store-operated calcium entry channels in human platelets. Mol. Pharmacol. 60, 541-552 (2001).

25. Harteneck, C. \& Gollasch, M. Pharmacological modulation of diacylglycerolsensitive TRPC3/6/7 channels. Curr. Pharm. Biotechnol. 12, 35-41 (2011).

26. Wei, M. et al. Molecular mechanisms underlying inhibition of STIM1-Orai1mediated Ca2+ entry induced by 2-aminoethoxydiphenyl borate. Pflügers Arch. 468, 2061-2074 (2016).

27. Gilio, $\mathrm{K}$. et al. Functional divergence of platelet protein kinase $\mathrm{C}(\mathrm{PKC})$ isoforms in thrombus formation on collagen. J. Biol. Chem. 285, 23408-23417 (2010).
28. Littlechild, R., Zaidman, N., Khodaverdi, D. \& Mason, M. J. Inhibition of KCa3.1 by depolarisation and 2-aminoethoxydiphenyl borate (2-APB) during Ca2+ release activated $\mathrm{Ca} 2+(\mathrm{CRAC})$ entry in human erythroleukemia $(\mathrm{HEL})$ cells: implications for the interpretation of 2-APB inhibition of CRAC entry. Cell Calcium 57, 76-88 (2015).

29. Wolfs, J. L. et al. Reversible inhibition of the platelet procoagulant response through manipulation of the Gardos channel. Blood 108, 2223-2228 (2006).

30. Jing, W. et al. Calpain cleaves phospholipid flippase ATP8A1 during apoptosis in platelets. Blood Adv. 3, 219-229 (2019).

31. Zhou, B. D., Guo, G., Zheng, L. M., Zu, L. Y. \& Gao, W. Microparticles as novel biomarkers and therapeutic targets in coronary heart disease. Chin. Med. J. 128, 267-272 (2015).

32. van der Pol, E. \& Harrison, P. From platelet dust to gold dust: physiological importance and detection of platelet microvesicles. Platelets 28, 211-213 (2017).

33. Owens, A. \& Mackman, N. Microparticles in hemostasis and thrombosis. Circ. Res. 108, 1284-1297 (2011).

34. Morel, O., Jesel, L., Freyssinet, J. M. \& Toti, F. Cellular mechanisms underlying the formation of circulating microparticles. Arterioscler. Thromb. Vasc. Biol. 31 15-26 (2011).

35. Diver, J. M., Sage, S. O. \& Rosado, J. A. The inositol trisphosphate receptor antagonist 2-aminoethoxydiphenylborate (2-APB) blocks Ca2+ entry channels in human platelets: cautions for its use in studying Ca2+ influx. Cell Calcium 30, 323-329 (2001).

36. Harper, M. T. \& Poole, A. W. PKC inhibition markedly enhances Ca2+ signaling and phosphatidylserine exposure downstream of protease-activated receptor1 but not protease-activated receptor-4 in human platelets. J. Thromb. Haemost. 9, 1599-1607 (2011).

37. Harper, M. T. et al. Transient receptor potential channels function as a coincidence signal detector mediating phosphatidylserine exposure. Sci. Signal. 6, ra50 (2013)

38. Harper, M. T. \& Poole, A. W. Store-operated calcium entry and non-capacitative calcium entry have distinct roles in thrombin-induced calcium signalling in human platelets. Cell Calcium 50, 351-358 (2011).

39. Dellis, O., Mercier, P. \& Chomienne, C. The boron-oxygen core of borinate esters is responsible for the store-operated calcium entry potentiation ability. BMC Pharmacol. 11, 1 (2011).

40. Baldwin, A. G. et al. Boron-Based Inhibitors of the NLRP3 Inflammasome. Cell Chem. Biol. 24, 1321-1335.e5 (2017).

41. MacKenzie, A. et al. Rapid secretion of interleukin- $1 \beta$ by microvesicle shedding Immunity 15, 825-835 (2001).

42. Flaumenhaft, R. in Platelets and Megakaryocytes 273, 365-378 (Humana Press, 2004).

43. Chokshi, R., Fruasaha, P. \& Kozak, J. A. 2-Aminoethyl diphenyl borinate (2-APB) inhibits TRPM7 channels through an intracellular acidification mechanism. Channels 6, 362-369 (2012).

44. Jardín, I., López, J. J., Pariente, J. A., Salido, G. M. \& Rosado, J. A. Intracellular calcium release from human platelets: different messengers for multiple stores. Trends Cardiovasc. Med. 18, 57-61 (2008).

45. Sage, S. O., Pugh, N., Mason, M. J. \& Harper, A. G. S. Monitoring the intracellular store $\mathrm{Ca} 2+$ concentration in agonist-stimulated, intact human platelets by using Fluo-5N. J. Thromb. Haemost. 9, 540-551 (2011).

46. Wei, H. \& Harper, M. T. ABT-737 triggers caspase-dependent inhibition of platelet procoagulant extracellular vesicle release during apoptosis and secondary necrosis in vitro. Thromb. Haemost. 19, 1665-1674 (2019).

47. Schiro, A. et al. Endothelial microparticles as conveyors of information in atherosclerotic disease. Atherosclerosis 234, 295-302 (2014). 